\title{
Publicités multilingues : l'apport du traducteur en agence de communication marketing
}

Multilingual Advertising: the Translator's Contribution to a Communication and Marketing Agency

\section{Hugo Vandal-Sirois}

\section{(2) OpenEdition}

\section{Journals}

Édition électronique

URL : http://journals.openedition.org/ilcea/1106

DOI : 10.4000/ilcea.1106

ISSN : 2101-0609

Éditeur

UGA Éditions/Université Grenoble Alpes

\section{Édition imprimée}

ISBN : 978-2-84310-203-5

ISSN : 1639-6073

Référence électronique

Hugo Vandal-Sirois, «Publicités multilingues : I'apport du traducteur en agence de communication marketing », ILCEA [En ligne], 14 | 2011, mis en ligne le 30 juin 2011, consulté le 01 mai 2019. URL: http://journals.openedition.org/ilcea/1106; DOI : 10.4000/ilcea.1106

Ce document a été généré automatiquement le 1 mai 2019.

(c) ILCEA 


\section{Publicités multilingues : l'apport du traducteur en agence de communication marketing}

Multilingual Advertising: the Translator's Contribution to a Communication and Marketing Agency

Hugo Vandal-Sirois

Depuis les toutes premières représentations de saint Jérôme, le traducteur possède l'image d'un être reclus qui effectue sa besogne emmuré dans ses ouvrages de référence. Ce stéréotype est encore bien présent aujourd'hui, alors que le traducteur se situe souvent à l'extrémité d'une longue chaîne de production et demeure parfois un simple sous-traitant plutôt qu'un coéquipier ou un partenaire en qui on peut avoir confiance. Pour ses clients et ses collègues, son expertise est réduite aux questions purement linguistiques et ses services sont perçus comme un mal nécessaire. Heureusement, il existe certains secteurs d'activité où les compétences du traducteur sont utilisées à bien meilleur escient. Non seulement son apport est valorisé, mais son implication dans le processus de production est considérée comme bénéfique, voire indispensable. Les projets de traduction ancrés dans les domaines artistiques et culturels favorisent particulièrement ce type de travail collaboratif, et le cas de l'adaptation publicitaire est très éloquent. Peu à peu, les traducteurs intègrent les équipes de création et de production des agences de publicité, et sont invités à collaborer avec les différents acteurs impliqués dans le développement d'une campagne publicitaire multilingue. Cette dynamique d'échange et de proximité permet au traducteur de bénéficier d'une plus grande marge de manœuvre pour trouver des solutions créatives et efficaces aux problèmes traductionnels de tout ordre, et de produire un document d'arrivée dont la cohésion avec les objectifs des clients et partenaires est totale. Cet article propose donc d'étudier la relation entre le traducteur publicitaire et son milieu de travail au sein d'une équipe multidisciplinaire, ainsi que la façon dont ses différents collègues influencent son travail et augmentent son efficacité. 
2 D'emblée, il faut reconnaître que l'idée du traducteur impliqué dans un processus créatif qui va au-delà du transfert linguistique est loin d'être exclusif à la publicité et au marketing. Au théâtre, par exemple, le traducteur peut être appelé à travailler de concert avec le metteur en scène afin de participer activement à la création d'un projet artistique cohérent. Il assiste alors aux répétitions, discute des solutions et des stratégies de traduction retenues et effectue des modifications spontanées sur le terrain, en observant le jeu des acteurs qui verbalisent son œuvre. L'échange avec les auteurs des textes originaux fait même partie intégrante de la méthode de travail de Linda Gaboriau, traductrice renommée de pièces québécoises vers l'anglais. À ce propos, elle écrit :

[...] je traduis des œuvres d'auteurs contemporains. Auteurs que je peux rencontrer et mieux connaître. Au même titre, la fréquentation assidue du théâtre québécois nourrit sans aucun doute mes traductions. Je peux me référer à la création d'une pièce, revivre ses intonations, ses rythmes, ses émotions et son impact sur le public d'ici. (Gaboriau, 1990, p. 46)

3 Cette participation procure donc au traducteur des ressources qui lui permettront de définir un postulat traductif précis et d'explorer avec confiance des pistes de solutions difficilement envisageables lorsqu'il n'a que le texte original comme outil de travail. C'est toutefois dans la foisonnante industrie de l'adaptation publicitaire que l'intégration du traducteur aux équipes de création et la mise en commun de différentes compétences s'avèrent les plus efficaces. Cette situation est particulièrement intéressante à étudier aujourd'hui, alors que les modes de communication des entreprises et organismes multinationaux connaissent, depuis quelques années, une période de bouleversements qui a changé non seulement le travail du traducteur, mais également son rôle dans la chaîne de production des communications commerciales et institutionnelles.

\section{Le marché de l'adaptation publicitaire et l'apport du traducteur}

4 Afin de mieux saisir la nature de ces changements, il importe de s'arrêter brièvement sur l'évolution du contexte socioéconomique de la traduction publicitaire, puis sur ses caractéristiques. D'abord, la mondialisation, l'expansion d'Internet, ainsi que la prolifération du commerce en ligne et des réseaux sociaux multiplient les occasions et surtout les besoins de communication interculturelle. Plus il y a de contenants, plus il faut de contenu. Depuis la fin des années 1990, les stratèges en marketing des grandes sociétés perçoivent la planète comme un village global, dont les habitants ont des goûts, des besoins et des comportements de consommation de plus en plus semblables. Il n'est plus rare qu'un seul et unique message soit véhiculé à l'échelle planétaire, à quelques ajustements près. Cela signifie, il va sans dire, que dans les marchés périphériques, les mandats d'adaptation ne cessent de s'accroître. Alors qu'auparavant, on n'hésitait pas à confier la création de différentes campagnes aux agences locales, on leur demande désormais d'adapter un message unique souvent conçu par les grandes agences de Paris, New York, Toronto, Londres ou de la Californie, par exemple. Dans le présent contexte socioculturel mondial, cette tendance à favoriser la centralisation des activités de communication s'explique également par des facteurs commerciaux. Adapter une publicité plutôt que concevoir un nouveau message pour chaque culture visée entraîne d'importantes économies d'échelle car, normalement, l'opération devrait être plus rapide et surtout nécessiter moins de ressources humaines et matérielles. Toutefois, un second 
avantage non négligeable est certainement que la diffusion d'un message unique permet un contrôle bien plus serré de l'image d'une entreprise et de ses communications institutionnelles qu'avec une multitude de sous-traitants aux quatre coins de la planète qui conçoivent chacun leur propre message.

5 Ainsi, certaines agences de communication constatent malgré elles des changements importants dans les mandats qui leur sont confiés, ce qu'elles ne voient pas nécessairement d'un bon œil. Les créateurs, experts en stratégies marketing, en psychologie de la communication et en rédaction, sont alors confrontés à des problèmes de transfert linguistique et culturel pour lesquels ils sont peu outillés, formés et expérimentés. C'est donc aux traducteurs et non plus aux rédacteurs et autres experts que revient la responsabilité de rédiger le contenu des messages présentés dans la culture cible et de prendre des décisions très importantes quant aux ajustements à effectuer par rapport au contenu du message original. Il faut toutefois ajouter que certains professionnels de la publicité hésitent à faire appel aux services d'un traducteur, car ils craignent d'obtenir une version plutôt terne, sans la vitalité et la force que l'on retrouve habituellement dans les textes argumentatifs et promotionnels. Ils peuvent alors s'improviser eux-mêmes traducteurs, avec un succès inégal. Comme dans bien des domaines de spécialité, c'est justement la rareté des traducteurs professionnels qui possèdent une plume publicitaire efficace et une connaissance approfondie de la publicité et des différents médias qui fait leur valeur, d'autant plus que la formation dans ce domaine semble n'occuper qu'une place marginale dans les programmes universitaires.

Quoique récente, l'évolution du rôle du traducteur publicitaire intéresse déjà les traductologues. Dans son livre La Communication multilingue (2008) par exemple, Mathieu Guidère en dresse sommairement les grandes lignes, qu'il divise en trois phases distinctes façonnées principalement par les limites du champ d'intervention du traducteur publicitaire. Il est à noter que ces trois phases ne suivent pas une chronologie universelle précise, mais plutôt une suite de changements marqués dans la perception du traducteur et de ses compétences. Encore aujourd'hui, les différents marchés, agences et clients ne se situent pas tous au même stade. D'abord, le traducteur est perçu comme un simple exécutant, le dernier maillon d'une longue chaine. Il doit traduire des textes qui ont été extraits de leur contexte avec trop peu de documentation, parfois même sans voir les images qui accompagnent le texte, et dans des délais souvent sous-estimés par ses clients et ses collègues. Ensuite, dans la seconde phase, on se rend compte que le temps et l'énergie consacrés à la présentation du contexte de communication au traducteur constituent en fait un investissement qui rehausse la qualité et l'efficacité du produit fini. Petit à petit, le traducteur fait sa place dans les équipes de spécialistes et accède à l'ensemble des éléments dont il a besoin, dont bien sûr les éléments visuels de la publicité. À ce stade, les clients et collègues du traducteur comprennent déjà mieux les enjeux et les risques de la communication interculturelle, ainsi que l'indéfectible lien entre l'image et le texte.

7 Finalement, la troisième phase correspond à l'acquisition par le traducteur du statut de conseiller sur la culture cible, en plus de celui d'expert en langues. Profitant de la confiance de ses collègues et clients, il jouit alors d'une plus grande liberté, et ses interventions ne modifient plus uniquement le texte, mais bien la publicité dans son ensemble. Il peut recommander de modifier les éléments graphiques, le ton, la teneur des arguments ou encore les références culturelles des messages publicitaires: "[Les traducteurs sont] non seulement dépositaires d'un savoir culturel vendeur, mais 
également détenteurs d'un savoir-faire technique susceptible de faciliter la recontextualisation du message [...]» (Guidère, 2008, p. 31). Les théoriciens du marketing Samuel Craig et Susan Douglas se sont également intéressés à l'importance de l'image dans la communication publicitaire, et par conséquent à la nécessité d'en proposer des adaptations pour les nouveaux publics cibles, au même titre que le texte.

Les instruments verbaux et non verbaux doivent tous deux être traduits afin d'être utilisés dans des contextes linguistiques et culturels différents. Bien que la nécessité de traduire les instruments verbaux soit généralement reconnue et que les exemples d'erreurs causées par des traductions erronées abondent, le besoin de traduire les stimuli visuels est habituellement bien moins reconnu. Il est néanmoins important de comprendre que les stimuli visuels ne sont pas nécessairement universels ou panculturels ${ }^{1}$. (Notre traduction, Craig et Douglas, 2005, p. 254).

Il faudrait toutefois ajouter que cette évolution en trois phases dont l'aboutissement correspond à la reconnaissance du traducteur comme expert multidisciplinaire en communication dépend autant des clients et des directeurs d'agence de publicité que des traducteurs eux-mêmes. C'est à la longue que les traducteurs peuvent démontrer l'étendue de leurs compétences et leur capacité à produire une adaptation qui possède toutes les qualités formelles et fonctionnelles d'un message conçu sur mesure pour le nouveau public cible, tout en conservant l'effet recherché par l'annonceur. Lorsque le traducteur aura gagné la confiance de ses collègues ou employeurs, non seulement il profitera d'une marge de manœuvre plus importante pour trouver des solutions aux différents problèmes traductionnels, mais il se forgera également une place qui lui est propre parmi les équipes de création bien ficelées des agences. Il contribuera ainsi au développement d'un milieu de travail collaboratif, ergonomique, favorable à l'inventivité et adapté à cette hausse des besoins de communications corporatives multilingues et des mandats d'adaptation publicitaire. Cependant, afin de bien saisir les mécanismes de son intégration et le rôle qu'il jouera au sein d'une équipe de spécialistes du marketing, de la rédaction, de la consommation, de l'image, de la psychologie et des médias, il est essentiel de rappeler les spécificités de la traduction publicitaire.

\section{Fonctions et aspects théoriques de la traduction et de l'adaptation publicitaire}

9 La publicité est l'un des rares domaines où l'approche cibliste fait l'unanimité, tant chez les traductologues que chez les praticiens. Afin de favoriser la réception d'une publicité étrangère chez un lectorat précis, il est d'emblée évident que le traducteur devra se distancer du texte original pour atteindre une conformité dans la culture cible. Le lecteur doit se sentir interpellé et se reconnaître dans le message, souvent en quelques secondes à peine. Par ailleurs, dans le même ordre d'idées, Roger Boivineau insiste sur la nature très créative de la traduction publicitaire, dans ce qui est certainement l'un des tout premiers articles de traductologie à traiter exclusivement de l'adaptation publicitaire :

L'adaptation consistera ainsi à écrire sur la trame suggérée par l'annonce originale un nouveau texte [...]. Là, il ne sera point besoin de respecter scrupuleusement la pensée de l'auteur, ni même son style. Il s'agira plutôt d'atteindre le but recherché avec l'original, et la voie pour rejoindre ce but pourra s'écarter sensiblement de celle suivie par le concepteur. (Boivineau, 1972, p. 15)

10 À la lumière de ces remarques sur les spécificités de l'adaptation publicitaire, complémentaires malgré un écart de plus de trente ans, il est facile d'établir un lien entre 
ce domaine de traduction spécialisé et certaines théories courantes en traductologie. Effectivement, la théorie du skopos (Vermeer, 1996) et les théories fonctionnalistes en général (Nord, 1997), qui incitent le traducteur à considérer prioritairement la fonction du texte (parfois appelé « la visée ») ainsi que ses lecteurs, correspondent à merveille aux mécanismes publicitaires. L'annonceur cherche à transmettre des informations à un public spécifique afin de modifier son comportement et ses opinions. Les concepteurs doivent donc s'interroger sur le public cible afin de déterminer ce qui l'intéressera au sujet du produit ou de la cause à promouvoir, les arguments auxquels il sera le plus sensible, le ton qui l'interpellera davantage, le contexte dans lequel il verra le message, etc. Bien entendu, le traducteur publicitaire devra impérativement effectuer le même travail de recherche et de réflexion que les créateurs du message car, pour des raisons sociales, économiques, culturelles, géographiques ou même religieuses, son public peut être très différent du public visé par l'original. C'est pourquoi l'objectif ne sera pas d'être littéralement ou sémantiquement fidèle au texte, ni même loyal envers l'auteur, mais plutôt de reproduire l'effet créé chez le lecteur du public de l'original. C'est justement cet effet qui laisse une impression marquante et qui assure l'efficacité du procédé publicitaire. Isabel Cómitre Narváez, qui s'intéresse beaucoup aux aspects culturels de la traduction, défend également cette approche cibliste axée sur la réception du message par le lecteur.

Toutes les modifications ou interventions réalisées par le traducteur sont justifiées afin d'obtenir le même effet dans la culture cible. Ces modifications, loin d'être des prises de liberté aléatoires, sont des options guidées par un souci de respect de l'univers de référence de la culture visée et de la fonction de traduction. (Cómitre Narváez, 2004, p. 88)

11 Une brève revue de littérature confirme par ailleurs que l'adaptation est la norme en traduction publicitaire. Georges Bastin écrit même que l'adaptation est indispensable, car elle rétablit «un équilibre communicationnel rompu par la traduction» (Bastin, 1993, p. 447). Il ajoute que dans certains domaines, et c'est certainement le cas en publicité, refuser de recourir à l'adaptation, qu'elle soit ponctuelle ou globale, produit des textes d'arrivée qui informent du vouloir-dire de l'original, mais en négligent la visée. Cela peut aller jusqu'à la production d'une version cible terne, inefficace ou carrément inutile. Comme le traducteur devra, à différents degrés, s'éloigner de la publicité originale, celleci ne constituera donc qu'une partie de l'ensemble des éléments pris en considération pour déterminer une stratégie d'adaptation globale.

Idéalement, le traducteur publicitaire se voit accorder toute la liberté et l'autonomie dont il a besoin pour effectuer ces interventions et n'a pas à se justifier continuellement auprès d'un client unilingue craintif de reconnaître peu d'éléments de sa publicité originale. Cette réaction est par ailleurs normale au début d'une nouvelle relation de travail, dans la mesure où le client ne possède pas les connaissances culturelles et linguistiques pour évaluer la qualité de l'adaptation. Heureusement, la nécessité de prioriser la finalité d'un texte pendant son adaptation ne fait pas l'unanimité que chez les traducteurs et les traductologues, mais également chez les autres experts de la communication institutionnelle et publicitaire. Dans un ouvrage marquant sur le marketing international, Michael Czinkota et Ilkka Ronkainen avancent même que pour évaluer la qualité d'une adaptation publicitaire, il faut mesurer l'impact créé chez les lecteurs de la culture cible (Czinkota et Ronkainen, 2007, p. 59). Pour parvenir à produire une adaptation aussi frappante, drôle, touchante et mémorable que l'original, ce que Boivineau appelle une «belle efficace » (Boivineau, 1972, p. 15), en référence au célèbre courant français de 
traduction cibliste, l'intégration du traducteur dans une équipe de concepteurs et de spécialistes de la communication est primordiale. Il se retrouve ainsi dans un environnement créatif stimulant qui lui donne accès à une variété d'outils et de documents nécessaires pour justifier certaines initiatives et, peut-être plus important encore, lui permet d'apprendre et de développer ses aptitudes de rédacteur publicitaire et de stratège en marketing. De plus, comme la traduction publicitaire occupe souvent une place marginale dans les programmes universitaires, la possibilité de discuter avec des personnes-ressources et d'être entouré d'experts des différentes disciplines de la communication représente pour le traducteur une chance en or de se spécialiser dans un domaine où la demande croît sans cesse.

\section{Le traducteur au sein d'une agence de communication et de marketing}

Règle générale, les traducteurs ne sont pas experts des médias, ni des relations publiques, ni de l'image, ni de la rédaction publicitaire. À l'instar des autres domaines de la traduction spécialisée, ils pourront découvrir les rudiments de la rédaction publicitaire et même de la création en poursuivant leurs études dans un domaine lié à la communication, au marketing ou à la consommation, ou encore en analysant les bons et les mauvais coups des traducteurs qui ont travaillé sur des campagnes semblables. Toutefois, c'est en plongeant au cœur de l'action qu'ils maîtriseront leur art. Dans le milieu dynamique et hétéroclite de la création publicitaire, où le talent, la compétence et l'expérience pèsent plus lourd dans la balance que tout diplôme, le principal conseil que les directeurs de la création donnent aux traducteurs novices se résume ainsi : «C'est en forgeant que l'on devient forgeron. » En évoluant au sein d'une agence de publicité qui reconnaît l'étendue de ses compétences, le traducteur travaillera sur un pied d'égalité avec les autres éléments de l'unité créative et pourra participer activement non seulement aux opérations de transferts langagiers, mais éventuellement à l'élaboration des études de marché, aux stratégies de communication et aux campagnes publicitaires elles-mêmes. Grâce à une structure d'entreprise efficace, qui favorise la collaboration entre les différents professionnels et l'échange d'information, chaque membre de l'équipe sera en mesure d'inspirer et d'alimenter la réflexion du traducteur, ou encore de le conseiller et de l'orienter vers des pistes de solutions lorsqu'il est confronté à différents problèmes traductionnels ou à des contraintes particulières. Pour un traducteur généraliste qui effectue des contrats d'adaptation publicitaires à la pige et surtout à distance, cette nouvelle intégration physique entraîne de grands changements dans son environnement et ses habitudes de travail. Il s'avère donc intéressant d'étudier le lien qui lie le traducteur aux autres experts de l'agence et l'influence que ceux-ci ont sur l'opération traductionnelle dans son ensemble. Pour y parvenir, nous retiendrons la structure d'une agence type de taille moyenne, qui offre des services de création et de rédaction (développement d'une image de marque, conception de campagnes, de slogans, de brochures, etc.), de production (infographie, tournage et enregistrement de messages audiovisuels, sites Web) ainsi que de consultation et de diffusion (plans de communication, élaboration de stratégies, gestion des médias). Vu la nature des opérations de communication multilingue ou interculturelle, il n'est pas rare que les activités du traducteur couvrent ces trois catégories de services. Les agences distinguent parfois les services d'adaptation des services de traduction, afin que les clients obtiennent 
ce dont ils ont besoin et ne soient pas surpris des initiatives proposées par les traducteurs. Les clients qui tentent une première percée dans une culture étrangère ne sont pas nécessairement familiers avec les particularités de la communication multiculturelle et doivent comprendre les raisons qui poussent les traducteurs à adopter une stratégie traductionnelle plutôt qu'une autre. Les occurrences d'adaptation et même de (re)création seront ainsi plus courantes dans le discours argumentatif, souvent lié à la culture, aux émotions et à l'humour que dans les passages davantage descriptifs, techniques ou juridiques (les conditions pour profiter d'une offre promotionnelle, les détails d'un concours ou encore les sections plus techniques d'une brochure d'un produit de haute technologie, par exemple).

\section{Création}

Habituellement composées de concepteurs et de rédacteurs sous la supervision d'un directeur artistique, les unités créatives connaissent non seulement le client et son produit, mais également les intérêts et les spécificités du public qu'il souhaite toucher. C'est avec ces experts, nommés les « créatifs » dans le milieu, que le traducteur décidera quels aspects du message original devront être modifiés, supprimés ou remplacés, et quels ton et registre de vocabulaire seront les plus appropriés pour le public en question. Ces choix peuvent relever de la simple synonymie (notre ordinateur portable est-il solide, robuste, dur comme du roc?) ou du registre de la langue. Donnons l'exemple assez courant du titre Spring sale qui, selon la marque et son public, pourra être traduit par « solde printanier » ou par « vente du printemps »... n'en déplaise à certains! De plus, les décisions d'adaptation plus importantes, en cas d'incohérence ou de rupture entre la culture de départ et celle d'arrivée, par exemple, seront elles aussi souvent prises en collaboration avec les créatifs. Le traducteur n'est donc plus laissé à lui-même lorsqu'il est confronté à des difficultés ou à des dilemmes. Comme il est de plus en plus appelé à travailler au lieu de création même, il pourra discuter, d'égal à égal, des possibilités d'adaptation envisageables. Il peut s'agir notamment d'apporter des ajustements lorsque l'on craint que les arguments originaux n'aient pas le même impact chez le lecteur, comme certains types d'humour, les références aux mouvements de masse telles que «tout le monde le fait, pourquoi pas vous? ", à l'argent, ou tout simplement pour des raisons de valeurs ou de réalités différentes. Pensons à une publicité pour une gamme de motocyclettes que nous avons dû adapter pour les consommateurs québécois. Ce message, conçu à Toronto, affirmait que les paiements mensuels que doivent effectuer les acheteurs étaient inférieurs au prix d'un forfait mensuel de transport en commun. Toutefois, comme le prix de ces laissez-passer est moins élevé dans les grandes agglomérations urbaines du Québec qu'à Toronto, l'argument perdait tout son poids. Il a donc fallu créer un nouveau référent qui correspondait au cadre original de la publicité. Cet exemple, où conserver le sens de l'original serait incompatible avec la fonction du message, illustre à la fois la nécessité de l'adaptation en publicité et également l'importance pour le traducteur d'appartenir à la culture cible et de la connaître sous toutes ses coutures. C'est d'ailleurs pourquoi les adaptations des messages planétaires sont souvent effectuées localement, pour chaque culture cible.

Par ailleurs, il faut mentionner que, même s'il est entouré de rédacteurs professionnels, c'est très souvent le traducteur qui se chargera de rédiger le nouveau texte. Même en modifiant le contenu du message, le traducteur doit s'assurer que, au minimum, l'identité 
de l'annonceur et son image de marque soient respectées. La qualité créative du travail du traducteur publicitaire, de plus en plus reconnue, n'est donc assurément pas étrangère à son intégration dans les équipes de concepteurs et aux libertés de plus en plus importantes qu'il se voit accordées. À ce sujet, Roger Boivineau est sans équivoque. Dans une table ronde, il avance que, outre les compétences purement linguistiques, « il devrait y avoir peu de différence entre un bon concepteur publicitaire et un bon adaptateur publicitaire " (Paré et al., 1972, p. 41). Évoluer à proximité de créatifs permet ainsi au traducteur d'approfondir sa maîtrise des subtilités du langage publicitaire. De plus, si les créatifs de l'agence effectuent des mandats pour un client depuis un certain temps, ils pourront procurer au traducteur une grande variété de documents qui lui permettront d'établir un postulat traductif juste et précis, puis de prendre les meilleures décisions quant aux stratégies à adopter. L'ensemble des publicités traduites et diffusées précédemment, une analyse des principaux concurrents présents dans le marché ciblé, des études de marché, et même des rapports de groupes de discussion (focus groups) sont des exemples d'outils de travail essentiels qui orientent et exercent une grande influence sur l'opération d'adaptation, et sur lesquels le traducteur pigiste, non intégré à l'agence, peut rarement mettre la main.

\section{Production}

16 Le traducteur pourra également tirer profit d'une collaboration avec les experts de l'image et de la communication visuelle. L'interdépendance entre l'illustration et le texte joue souvent un rôle clé dans la perception d'un message publicitaire, et s'avère parfois essentielle pour susciter des émotions fortes ou un effet dont le public se souviendra. Le traducteur qui ne reçoit qu'un texte extrait de son ensemble, que ce soit pour des raisons pratiques ou encore parce que son client ignore ce qu'implique réellement l'acte traduisant, ne dispose pas de tous les outils requis pour bien faire son travail. De plus, le traducteur est un langagier et ne maîtrise pas nécessairement les codes de l'image, des couleurs ou de l'iconographie. Jeremy Munday note d'ailleurs que « ce lien entre le visuel et l'image est crucial et requiert bien plus d'analyses que ce qui a été fait à ce jour en traductologie ${ }^{2}$ " (2004, p. 211). Ainsi, lorsqu'ils sont confrontés à un problème de traduction lié à l'image (d'ordre graphique, spatial, typographique, culturel ou sémantique), les traducteurs en agence pourront explorer des pistes de solution qui leur étaient difficilement accessibles auparavant, pour des raisons d'expertise technique notamment. En étant sur place, le traducteur pourra s'assurer que son texte est bien intégré à la publicité et trouver des solutions efficaces lorsque l'adaptation du contenu visuel de la publicité est requise pour reproduire l'effet de l'original chez le public cible. Il peut s'agir, par exemple, d'un ajustement des repères géographiques qui permettent aux lecteurs de se reconnaître dans la publicité (en remplaçant les dunes du désert de l'Arizona par une forêt boréale), ou encore de la suppression d'un élément graphique qui n'évoquera pas les mêmes émotions chez les lecteurs des deux cultures différentes. Pensons notamment à la feuille d'érable, symbole du fédéralisme canadien par excellence, qui disparaît comme par magie des bouteilles de bière Labatt Bleu et des tasses de la chaîne de café Tim Horton's distribuées au Québec. Ces modifications graphiques, souvent suggérées par le traducteur et réalisées par un infographiste, favorisent la réception et l'efficacité des messages auprès du public cible et conservent davantage la fonction de l'original que lorsque seuls les mots sont adaptés. En plus de réduire les risques d'erreurs (accents disparus, césures dans les mots, disposition douteuse du texte, etc.), assez élevés 
lorsque le texte adapté est inséré à distance par un infographiste qui ne parle pas la langue d'arrivée, cette collaboration entre experts de la langue et de l'image permet de gagner un temps précieux, car les délais alloués par les clients sont souvent restreints. Bref, en raison de la réorganisation de l'espace de travail du traducteur publicitaire, ce dernier hérite parfois d'un mandat d'assurance de la qualité du produit final qui va bien au-delà la traditionnelle étape de la révision linguistique.

En outre, les messages publicitaires prennent plusieurs formes, et le traducteur est souvent impliqué dans la production de messages multimédias. Il est alors confronté aux contraintes de la traduction audiovisuelle, qui s'ajoutent aux difficultés du discours publicitaire. Lorsqu'il doit travailler sur l'adaptation d'un message télévisuel ou radiophonique, le traducteur adapte un scénario qui ne sera pas un produit final, mais bien un outil de travail pour un réalisateur, des comédiens, un directeur de plateau, etc. Sa connaissance de la culture cible l'amènera parfois à participer aux séances de casting et même à se présenter en studio ou sur le plateau pour orienter le jeu des acteurs qui « donnent vie » aux dialogues et aux narrations qu'il a écrits. Cette manière spontanée de travailler, qui n'est pas sans rappeler certaines méthodes de traduction théâtrale, place le traducteur au centre d'un effort collectif qui exigera parfois un peu d'humilité, mais qui permet d'approfondir ses suggestions et de leur donner une dimension nouvelle. En assistant à l'enregistrement de son adaptation en studio, en compagnie des acteurs, des producteurs et des techniciens, le traducteur acquiert des expériences pratiques du jeu et de la rédaction scénaristique qui lui seront très utiles tout au long de sa carrière, d'autant plus que ces notions n'ont certainement pas été abordées en profondeur durant sa formation.

18 Finalement, l'équipe de services interactifs (Web, médias sociaux, campagne en ligne, etc.) pourra former et aider le traducteur qui doit composer avec les contraintes graphiques et surtout techniques de la traduction de communications en ligne. Après tout, à l'image des rédacteurs, les traducteurs doivent apprendre à connaître les particularités de l'écriture pour le Web, comme la limite de 140 caractères de Twitter ou les habitudes de lecture des internautes, notamment. Les experts en nouvelles technologies de l'information et en marketing Web seront également des personnesressources en matière de terminologie spécialisée, et lorsque le traducteur doit effectuer des opérations de localisation (traduction spécialisée de logiciels, de sites Web et de produits de télécommunication). Effectivement, bien que cela soit moins fréquent que dans certains secteurs, comme l'informatique, les jeux vidéo ou l'administration, le traducteur publicitaire peut être appelé à faire de la localisation, notamment pour des logiciels promotionnels, des sites multimédias, des comptes de réseaux sociaux de sociétés ou des applications de téléphone intelligent.

\section{Consultation et diffusion}

19 Lorsqu'un client tente de transmettre des informations dans une culture qui lui est étrangère, il fait bien sûr appel au traducteur, qui produit une version d'arrivée conçue sur mesure pour assurer la meilleure réception possible chez le public visé (des points de vue linguistique, culturel, etc.). Toutefois, le processus communicationnel ne s'arrête pas là. Le message doit être diffusé au bon moment, au bon endroit et dans le bon contexte. Les spécialistes des médias, qui déterminent où et à quelle fréquence le message produit par le traducteur sera présenté au public cible, peuvent eux aussi avoir une grande 
influence sur l'opération de traduction. En plus des facteurs liés à la marque et aux caractéristiques du public cible, le traducteur devra adapter son style et son niveau de langage à l'espace publicitaire prévu pendant l'élaboration de la stratégie de communication. Il faudra alors considérer la nature du média (imprimé, sonore, en ligne, etc.) et son style (publication spécialisée réputée, journal sensationnaliste à grand tirage, etc.). Pensons par exemple à la radio, un média d'accompagnement auquel l'auditeur porte rarement toute son attention, ou encore au panneau publicitaire au bord de l'autoroute, qui doit être lu et compris en un clin d'œil. D'ailleurs, les études de marché effectuées par les experts en consommation et en marketing permettront au traducteur d'identifier des écarts importants entre les cultures source et cible, qui pourraient poser obstacle à la communication. Ainsi, le contenu d'une publicité pour un produit bien connu dans la culture source ne peut être le même dans une culture où ce produit est une nouveauté. C'est alors souvent au traducteur, parfois en collaboration avec un rédacteur, d'adapter le message en considérant que le public cible possède un savoir bien différent de celui du public visé par les concepteurs de la publicité originale. Les archives de l'agence et surtout les documents de marketing fournis par le client deviennent dans ce cas des outils très précieux, dont le traducteur contractuel œuvrant à l'externe doit généralement se passer pour des raisons de confidentialité.

\section{Direction artistique}

Toute opération de communication se déroule sous la supervision d'un directeur artistique, qui a le dernier mot sur toutes les décisions créatives suggérées par les membres de l'équipe, ce qui inclut bien entendu les traducteurs. Ce D.A. (encore le jargon du milieu!) connait le client et ses produits sur le bout des doigts et est la personneressource par excellence pour orienter le traducteur et lui fournir de précieux renseignements tout au long du processus traductionnel. De plus, si le traducteur publicitaire expérimenté peut avoir la certitude que son travail sera efficace dans la culture cible et que l'effet produit par le message original a été préservé, le D.A., lui, est en mesure de déterminer si cette traduction correspond au client, au produit et à l'image de marque.

\section{Conclusion}

21 Ainsi, après un tel tour d'horizon de l'environnement de travail du traducteur publicitaire intégré dans une agence, il parait évident qu'un traducteur travaillant en solo et isolé au bout d'une chaîne de production ne dispose pas de tout ce dont il a besoin pour prendre les meilleures décisions et produire la meilleure traduction possible. En plus des ressources matérielles importantes, les traducteurs en agence de publicité profitent des compétences et des talents variés des spécialistes, qui deviennent de véritables coéquipiers, grâce entre autres à l'organisation spatiale du bureau et à la formation d'unités de travail qui favorisent habituellement la collaboration et la complicité. Cette intégration dans un environnement créatif qui, rappelons-le, est plutôt récente, leur permet d'approfondir leur maitrise du discours publicitaire afin de s'éloigner parfois de l'original et de prendre des initiatives éclairées qu'ils pourront aisément justifier auprès du client. Ce dernier point est particulièrement crucial en cette ère de mondialisation, où le besoin d'échanger de l'information entre les différentes cultures croît sans cesse et où 
le traducteur est, bien sûr, expert des langues, mais d'abord et avant tout communicateur.

D'une certaine façon, l'intégration du traducteur au sein des agences peut être perçue comme une reconnaissance de l'importance de son travail et de l'étendue de ses compétences. Grâce à la pertinence de leur contribution, ainsi qu'à une situation socioéconomique planétaire plus que favorable, admettons-le, ils sont nombreux à être passés de simple fournisseur à membre en bonne et due forme des agences de publicité. L'univers du marketing illustre bien comment ils peuvent travailler en équipe et contribuer à un effort collectif dont le transfert linguistique n'est qu'un élément d'une vaste opération de communication. Toutefois, il serait aussi très intéressant d'étudier d'autres domaines de spécialisation où le travail d'équipe et la proximité de collègues aux formations différentes bonifient la qualité des traductions et en facilitent la production. En plus d'enrichir leurs connaissances sur les dynamiques du processus traductionnel, cela permettrait peut-être aux traducteurs de continuer à se distancer de ce sempiternel stéréotype d'être solitaire, eux qui pratiquent pourtant une activité essentielle à la communication interculturelle et, par le fait même, aux relations humaines.

\section{BIBLIOGRAPHIE}

BASTIN Georges L., « La notion d'adaptation en traduction », Meta : Journal des traducteurs, vol. 38, $\mathrm{n}^{\circ} 3,1993$, p. 473-478.

BOIVINEAU Roger, «L'A.B.C. de l'adaptation publicitaire », Meta :Journal des traducteurs, vol. 17, n $^{\circ}$ 1, 1972, p. 5-28.

Cómitre NARVÁEz Isabel, « Stratégies de traduction de l'ambivalence dans le texte publicitaire », Anales de filologia francesa, $\mathrm{n}^{\circ}$ 12, 2003-2004, p. 81-93.

CRAIG Samuel C. et Douglas Susan P., International marketing research, Chichester, John Wiley \& Sons, 2005, $482 \mathrm{p}$.

CZINKota Michael R. et RonKAInEN Ilkka A., International Marketing, Thomson/South-Western, $2007,646 \mathrm{p}$.

GABORIAU Linda, «Traduire le génie de l'auteur », Jeu : revue de théâtre, n 56, 1990, p. 43-48.

GUIDÈrE Mathieu, La communication multilingue, Bruxelles, De Boeck, 2008, 137 p.

MunDaY Jeremy, « Advertising: Some Challenges to Translation Theory », The Translator, vol. 10, $\mathrm{n}$ ० 2, 2004, p. 199-219.

NORD Christiane, Translating as a Purposeful Activity - Functionalist Approaches Explained, Manchester, St Jerome Publishing, 1997, 154 p.

PARÉ Marcel, BoIvineau Roger, Pelletier Jean-François, NoRMAndin Gérard et Roy Lucien, « Table ronde : l'adaptation publicitaire, oui ou non?", Meta: Journal des traducteurs, vol. 17, n 1, 1972, p. 29-46. 
VERMEER Hans J., A Skopos Theory of Translation, Heidelberg, Textcontext Wissenschaft, 1996, 136 p.

\section{NOTES}

1. Texte original : "Both verbal and nonverbal instruments need to be translated so that they can be used in different linguistic and cultural contexts. While the need for translation of verbal instruments is widely recognized, and examples of errors arising from mistranslation abound, the need for translation of visual stimuli is generally less well recognized. It is, nonetheless, important to realize that visual stimuli are not necessarily universal or pan-cultural. "

2. "This link between the visual and the word is crucial and requires far more analysis than has so far been offered in translation studies. "

\section{RÉSUMÉS}

Le traducteur a longtemps eu l'image d'un être reclus, qui effectue sa besogne avec comme unique compagnon sa panoplie d'ouvrages de référence. Encore aujourd'hui, il se situe fréquemment à l'extrémité de la chaîne de production et de diffusion des documents, et demeure un simple sous-traitant plutôt qu'un partenaire en qui l'on a confiance. Toutefois, dans certains domaines de spécialisation, l'implication du traducteur dans le processus créatif et sa collaboration avec les différents experts de la communication peuvent s'avérer très bénéfiques, et même indispensables. Le cas de l'adaptation publicitaire est particulièrement éloquent. Les traducteurs publicitaires sont de plus en plus intégrés aux équipes de création et de production des agences, et œuvrent désormais étroitement avec les directeurs artistiques, concepteursrédacteurs, réalisateurs, infographistes, etc. Ces échanges permettent au traducteur de bénéficier d'une plus grande marge de manœuvre pour trouver des solutions efficaces aux problèmes traductionnels de tout ordre, et de produire un document d'arrivée dont la cohésion avec les objectifs communicationnels des clients est totale. Cet article étudie le rôle du traducteur dans cette dynamique de travail collaboratif et la façon dont ces ressources influencent sa production puis bonifient son efficacité non seulement comme expert de la langue et de la culture cibles, mais également comme créateur à part entière.

For a long time, translators carried the image of recluse beings that work with their many reference books as only companions. Even to this day, they often are the very last link in the production chain of a document and regarded as simple suppliers instead of valued partners. However, in some specialized fields, the involvement of translators in the creative process and their collaboration with the various communication experts can turn out to be very beneficial, and even essential. The case of advertising adaptation is a good example. The advertising translators are increasingly integrated in the creation and production teams of the agencies, and thus work closely with the artistic directors, the copywriters, the directors, the computer graphics designers, etc. This cooperative environment gives the translators more flexibility in finding efficient solutions to any translation problems that may arise, and in creating a target document that will fully meet the client's communication objectives. This paper studies the role of the translators in this collaborative working environment and how these resources influence 
their production and increase their effectiveness not only as experts of the target language and culture, but also as creative agents in their own right.

INDEX

Keywords : adaptation, advertising, creativity, marketing, translation

Mots-clés : adaptation, créativité, marketing, publicité, traduction

\section{AUTEUR}

HUGO VANDAL-SIROIS

Université de Montréal 